\title{
EXTREMA ESCASEZ DE PAN EN ALICANTE: EL AÑ̃ 1333
}

\author{
Juan Manuel del Estal \\ Prof. de Historia Medieval \\ Universidad de Alicante
}

El año 1333 se tornó aciago también para la villa de Alicante, al igual que para otros muchos lugares de la Corona de Aragón, registrándose en sus Crónicas como "lo mal any primer» y muy particularmente en los anales del ignominado monje gerundés de Ullà, quien nos brinda un amplio relato de los males y azotes que el destino desató inmisericorde sobre las tierras y gentes del Ampurdán, exterminando casi su población campesina (1).

Escribe al respecto el citado historiador VILAR, al trazar los fundamentos económicos de las estructuras nacionales del Medievo catalán: «1333: - lo mal any primer-, aquest nom popular indica la consciència bastant clara d'un tomb entre uns temps considerats com a feliços $i$ una serie d'anys dramàtics. Aquest primer any o acte no fou una epidèmia sino una fam. Segons el Monjo d'Ullà, tot el baix poble del camp morí. A Barcelona hi ha cases buidades integrament i el nombre de morts devia ésser de 10.000. Per ajutjar l'episodi, certs detalls ens semblen més segurs que aquestes apreciacions globals. Hom no s'ha pogut inventar gratuitament que el 25 d'abril de 1333 una quartera de

(1) VILAR, Pierre, CATALUNYA DINS L'ESPANYA MODERNA. Recerques sobre els fonaments econòmics de les estructures nacionals. Vol. II: EL MEDI HISTORIC, Barcelona, $4 .^{\mathrm{a}}$ ed., 1973, pág. 147-148, con cita de PELLA I FORGAS, Josep, HISTORIA DEL AMPURDAN, II, págs. 650-670, donde reproduce parte de los Anales expresados. 
blat s'hagués venut a 42 lliures, una quartera d'hordi a 24 , i a 13 una quartera d'espelta. Ni que el mal hagi durat exactament dos mesos, fins a l'arribada a la capital de dos petits vaixells carregats de blat procedent de Tortosa i de quatre grans naus procedents de Sicilia, una de les quals era armada per la ciutat. D'altra banda, serà a partir d'aquesta data quan el Municipi barceloní s'encarrassarà a assegurar el proveïment en grans a tot preu" (2).

Aquel año 1333 fue uno, el primero, de una larga serie que, de mal en peor, se cebaría con sus lacerantes secuelas de carestía de vida, escasez alimentaria, hambre y peste, durante la mitad y último tercio del siglo XIV, en las gentes de la Corona de Aragón, a través de la tristemente célebre Peste Negra, con escalofriantes índices de mortalidad, al igual que en el resto de la población europea occidental (3).

A las plagas referidas del hambre y la peste hay que añadir además la de la guerra que por entonces (1330-1336) sostenía Aragón con la República de Génova, a consecuencia de la ocupación catalana de la isla de Cerdeña, iniciada ya por Jaime $\|$ el 1323, en busca fundamen-

(2) VILAR, Ibid.

(3) Ofrecemos bibliografía sumaria general y particular de España en las obras: VERLINDEN, Ch., "La grande peste de 1348 en Espagne", Revue belge de Philol. et Hist., XVII, 1/2, Bruselas 1938, págs. 103-146; CARPENTIER, E., "La peste noire: Famines et epidèmies au XIV siècle”, Anales. Economies. Soc. Civil, 1962, n. ${ }^{\circ}$, págs. 1.062-1.092, con mapa pág. 1.070; BIRABEN, J. N., "La peste dans l'Europe Occ. et le basin Mediterranien: principals epidèmies, conceptiones medicales, moyens de lutte", Le Concours Médical, 2 febr. 1963, págs. 781-790; RENOUARD, Yves, ETUDES D'HISTOIRE MEDIEVALE, Deuxième Partie: L'événement mondial le plus important du XIV siècle. LA PESTE NOIRE de 1348-1350, I, Paris 1968, págs. 143-155; II, págs. 157-164: Conséquences et interêt démographiques de la Peste Noire de 1348; LOPEZ DE MENESES, Amada, “Documentos acerca de la Peste Negra en los dominios de la Corona de Aragón", EEMCA, VI, Zaragoza, 1956, págs. 291-447 con transcripción de 157 dtos.; DOÑATE SEBASTIA, J. M. " "Datos negativos referidos a la Plana de Castellón en relación con la Peste Negra de 1348", VIII CHCA, II/1, Valencia 1969, págs. 27-45; KERN, H., "La peste negra y su influjo en la provisión de los beneficios eclesiást.", Ibid., págs. 71-85; KUCHLER, W., "La influencia de la peste negra sobre la Hacienda Real», Ibid., págs. 65-71; MARTINEZ MORELLA, V., "El Hospital de Peregrinos y enfermos de Alicante, a cargo de canónigos Antonianos", Ibid., págs. 85-95; MARTINEZ ORTIZ, J., "Una víctima de la peste: la Reina D. ${ }^{a}$ Leonor", Ibid., págs. 9-27; SANTAMARIA ARANDEZ, A., "La peste negra en Mallorca", Ibid., págs. 103-133; TRENCHS ODENA, J., "La archidióc. de Tarragona y la peste negra: Los cargos de la catedral", Ibid., págs. 45-65; WOLFF, Ph., "Reflexions sur les troubles sociaux dans les pays de la Couronne d'Aragon au XIV siècle", Ibíd., págs. 95-103; UBIETO ARTETA, A., CRONOLOGIA DEL DESARROLLO de la Peste Negra en la Península lbérica", Anexos de la Rev. HISPANIA, V, Madrid 1975, págs. 47-66; GRAU MONTSERRAT, M., "La peste negra en Mallorca", Bol. Soc. Castellonense de Cu/t., XLVI, n. ${ }^{\circ} 1,1970$, págs. 148-160; SOBREQUES CALLICO, J., "La peste negra en la Península Ibérica", Anuario de Est. Medievales, 7, Barcelona 1970/71, págs. 67-102; MAC NEILL, W. H., LE TEMPS DE LA PESTE. Assai sur les épidèmies dans l'histoire, Trad., París 1978. 
talmente de nuevos recursos cerealísticos sobre todo (4), al par de su situación estratégica singular para el dominio del Mediterráneo (5) y comercio ultramarino. Génova no podía ni quería por ello resignarse a la pérdida de aquel rico granero y desató en consecuencia una política de hostigamiento contra las naves catalanas que de regreso de Sicilia hacian alli escala o bien zarpaban de Cáller o de Yglesias, cargadas de grano con rumbo a Barcelona, debiendo enfrentarse a aquel corso genovés el almirante de la Corona allí desplazado, Francesc Carròs i de Cruilles (6).

Idéntica mala suerte corrian por lo común las embarcaciones de cabotaje que desde los puertos de Santa Pola, Alicante y Valencia se dirigían al Mediodía francés, como los bajeles, cocas y demás leños que se hallaban anclados en aquel litoral de la Corona. El acoso genovés se prolongó por todo el tiempo que duraron las hostilidades, hasta las Paz de Pedro IV el año 1336, haciéndose problemático en extremo el aprovisionamiento de cereales, procedentes de Sicilia y Cerdeña, como de cualquier otro punto del Mediterráneo (7).

(4) MUTGE, J., "Trigo sardo en Barcelona durante el reinado de Alfonso el Benigno", VIII CHCA, II/3, Valencia 1973, pág. 235: "La causa principal que movia a Jaime II a la conquista de Cerdeña era la situación estratégica de la isla, cuya posesión le permitiria señorear el Mediterráneo occidental. Otra causa que le llevó a conquistarla fue su importancia como fuente de recursos naturales. Es posible que Jaime viera en Cerdeña, 10 mismo que Pedro III había visto en Sicilia unos años antes, la solución del abastecimiento de Cataluña, fundamentalmente de las ciudades costeras, un granero propio donde proveerse y además una plaza o mercado al que llevar los productos propios"; SALAVERT Y ROCA, V., "Los motivos económicos de la conquista de Cerdeña", VI CHCA, Cerdeña 1957, págs. 437-445.

(5) VICENS VIVES, J., «La economía de los paises de la Corona de Aragón en la Baja Edad Media", VI CHCA, Cerdeña 1957, Madrid 1959, págs. 103-110.

(6) MITJA, M., "Barcelona y el problema sardo en el siglo XIV", VI CHCA, Madrid 1959, págs. 447-459, donde entre otras cosas afirma: "Génova montó una acérrima vigilancia frente a los puertos de Sicilia para impedir que cargamentos de trigo salieran para Barcelona...Galcerán Marquet, capitán de la escuadra barcelonesa, hizo proezas para romper el cerco genovés y acompañar con sus galeras las naves que habian de dirigirse a Barcelona. En 1333, a causa del hambre y la carestía del pan, los barceloneses se amotinaron y asaltaron las casas de los Consellers para obligarles a bajar el precio del trigo", pág. 451.

(7) MUTGE, J., "Trigo sardo en Barcelona...», l.c., pág. 237; vid. nota anterior; TANG" HERONI, M., ASPETTI DEL COMMERCIO DEI CEREALI NEI PAESI DELLA CORONA D'ARAGONA, 1. La Sardegna, Pisa 1981, pág. 83: "Durante la guerra tra l'Aragona e Genova (1330-1336), allorché vennero meno le possibilità di rifornimento cerealistico di Barcelona, accanto al grano prodotto dalle pianure di Urgell. Anche II vettovagliamento della flotta catalana fu assicurato quasi exclusivamente dal grano sardo... La disponibilità di eccedenze di cereali in Sardegna ancora all'inizio del 1333, delle quali non si riteneva di avere assoluto bisogno, é confermato dall'utilizzazione, allora avvenuta, di grano sardo per pagare debiti della corona con mercanti maiorchini. Ma inmediatamente dopo dovevano farsi sentire, anche in Sardegna, le conseguenze della dramatica situazione catalana di quel terribile mal any primer", págs. 85-86. 
Pero la guerra catalano-aragonesa no se limitó a repeler los ataques e incursiones piratas de naves genovesas, sino que amplió todavía más su campo de acción, al reanudar Alfonso IV contra el Reino de Granada la campaña militar por la conquista de Almería (1328-1334), repitiendo en vano la aventura que veinte años antes intentara realizar su padre, Jaime II, el año 1308, sin éxito alguno, empeñados ambos en el esfuerzo común de la expansión territorial de la Corona por "el quadrant Sud-est de la Península, l'antiga Taifa d'Almeria, desd'on podia exercir un control sobre les rutes de la Mànega Mediterrània, els accessos al'Estret $i$ els ports del Magreb Occidental» (8).

La fecha por tanto del 1333, como "el primer año malo" de las Crónicas catalanas, se enmarca plenamente en un período de profundas hostilidades de la Corona de Aragón con la República de Génova (1330-1336) y con el Reino nazarita de Granada (1328-1334), circunstancia y muy importante que ha de sumarse a las restantes de orden climático y epidémico, que originaron la terrible crisis económica, que tan profundamente perturbó las tierras y gentes de todo el litoral de la Corona (9), desembocando por último en la catastrófica Peste Negra y todas las secuelas derivantes en la segunda mitad del siglo XIV (10).

En este contexto histórico: social, político y económico, queremos presentar los documentos, que transcribimos íntegros en un Anexo documental, por hacer referencia explícita a la escasez extrema de pan, por que pasó la villa de Alicante en la segunda mitad de diciembre del año 1333, llegando a correr por ello se nos dice, gran peligro su población de tener que abandonar sus hogares y emigrar rápidamente a otro lugar, de no verse a tiempo sus vecinos aprovisionados debidamente de los cereales necesarios para su subsistencia.

Se trata de tres cartas del Infante D. Pedro, primogénito y sucesor de Alfonso IV de Aragón (Pedro IV el Ceremonioso), escritas dos de ellas en Valencia, el 17 de diciembre de 1333, con destino al bayle local de Cullera y al general del Reino de Valencia respectivamente, y la tercera, con fecha del 26 del mismo mes, al bayle general referido, Guillen Serani, interesándose vivamente por el socorro alimenticio inmediato a la villa

(8) SALRACH, J. M. ${ }^{2}$, HISTORIA DELS PAISOS CATALANS. Dels origens a 1714, 1, Barcelona 1981, pág. 540; DUFOURCQ, Ch. E., L'EXPANSIO ÇATALANA A LA MEDITERRANIA OCCIDENTAL. Segles XIII i XIV, trad., Barcelona 1969, pág. 310; VICENS VIVES, J., O.C. págs. 103-110; MITJA, o.c., págs. 450-452.

(9) ABADAL I VINYALS, R. D', Prólogo al vol. XIV de H. DE ESPAÑA de R. MENENDEZ PIDAL, ESPAÑA CRISTIANA, CRISIS DE LA RECONQUISTA. LUCHAS CIVILES por L. SUAREZ FERNANDEZ y JUAN REGLA CAMPISTOL, Madrid 1966, págs. XXVII-XXVIII; SALRACH, o.c., pág. 546.

(10) Vid. supra nota 3. 
de Alicante, debido a la gran escasez de pan que aflige a su población, ya que apenas puede hallarse a la venta en los establecimientos un pedazo que llevarse a la boca.

La primera está dirigida al bayle de Cullera para notificarle que le ha llegado una queja de los vecinos de Alicante contra él, por vedarles la licencia debida para zarpar con un barco, cargado de cereales, del puerto del lugar, con que abastecer la población tan falta de estos recursos alimenticios. Se trataba del cargamento de 150 cahices de panizo (caficia panicii) y otros tantos de sorgo o mijo (aderis seu adacie: adacça), trescientos en total, que una comisión de procuradores de la villa alicantina había podido comprar en Alcira, de acuerdo con la autorización expresa del Bayle General de la Reina D. ${ }^{a}$ Leonor de CastiIla, D. Guillen Serani, por el Reino de Valencia, y seguidamente habian hecho trasladar al puerto de Cullera, con el ánimo de embarcarlos allí con rumbo al destino indicado. La causa de todo ello, escribe el Infante, era la esterilidad extrema del año en curso, de modo que los citados vecinos no hallaban ya pan que llevarse a la boca en los establecimientos públicos "volentes providere evidenti necessitati et penurie frumenti, quia propter anni sterilitatem supraveniret loco predicto de Alacant, in quo panis coctus vix reperitur ut asseritur ad vendendum" (11), haciéndose cada día más apremiante la necesidad de evacuar sus hogares, por carecer de los recursos económicos indispensables para sobrevivir: "fame compulsi haberent deserere locum ipsum» (12).

Sin embargo, ante la negativa persistente del bayle local de Cullera a permitirles su exportación, llevado de un excesivo celo de mantener la prohibición real de sacar del citado puerto alguno de los productos de las consabidas "Coses vedades", entre las que figuraban en primer lugar los cereales, junto a caballos y armas, etc., D. Pedro de Aragón le ordena tajante que autorice inmediatamente la salida de su puerto del cargamento citado, sin estorbarlo lo más mínimo: "Quare...vobis dicimus et mandamus quatenus ipsis hominibus de Alacant vel eorum procuratori super extractionem dictorum CCC caficiorum panicii et adacie nullum impedimentum vel obstaculum apponatis" (13).

Ahora bien, deberá cuidar diligentemente que la cantidad extraída no sea en modo alguno superior a la permitida, trescientos cahices en total, y que todo el cargamento alimenticio sea transportado íntegramente a la villa de Alicante y no a otro lugar: "cavendo attente ne pretextu nos-

(11) ACA, Reg. 576, fol. 6 r., vid. Anexo Documental, I.

(12) Ibid.

(13) Ibid. 
tre concessionis huiusmodi inde ulla major frumenti seu rerum prohibitarum quantias extrahatur, quodque dicti homines de Alacant seu eius portator sufficienter caveant in posse vestro quod dicta kafficia panicii et adacie ad alias partes preterquam ad ipsum locum de Alacant minime defferantur» (14). La presente carta, añade el escribano, Pedro Jordani Durrer Expern, ante el estado pésimo de su primera redacción, fue destruida y redactada otra de nuevo, la que aparece registrada en el folio siguiente, de mano de Egidio Pérez (Petri) por orden del Infante (15). Termina el escrito recordando el Infante al bayle local que de cuanto queda dicho lo haga cumplir escrupulosamente con toda firmeza: "et hoc nullatenus inmutetis" (16).

La segunda carta lleva la misma fecha y va dirigida al Bayle General del Reino de Valencia, D. Guillen Serani, para referirle el grave estado alimenticio en que se encuentra la villa de Alicante y el acopio que ciertos procuradores suyos han podido llevar a cabo en Alcira, mediante la adquisición de trescientos cahices de trigo, con destino a aquella villa, pero que ante la negativa rotunda del bayle local de Cullera a dejar salir ningún producto cerealístico de su puerto, se ve por ello obligado a intervenir personalmente. Tras repetir las mismas causas de la escasez, que en la carta anterior, subrayando la extrema carencia de pan en los establecimientos, con que saciar el hambre reinante en la villa, manda al bayle general que ordene de inmediato al bayle local de $\mathrm{Cu}$ Ilera que otorge la licencia esperada de salida del cargamento en cuestión cuanto antes, para así poder satisfacer lo antes posible la necesidad tan perentoria de los vecinos de Alicante. Aquí se especifica que los procuradores alicantinos obtuvieron el preciado cargamento de los cobradores o recaudadores de las rentas de la Reina D. ${ }^{a}$ Leonor de Castilla, esposa del monarca Alfonso IV el Benigno, en la villa de Alcira y también en Cullera, arrojando la suma total de 40 cahices de panizo, 200 de sorgo o mijo (zahina, adacça), más 50 cahices de cebada, a base del diezmo adeudado a la referida señora, Su Majestad, la Reina de Aragón, doscientos noventa cahices en su totalidad, si bien en el resto de la carta se dé la cifra global de los trescientos (17): "vobis dicimus et mandamus quatenus ipsis hominibus de Alacant vel eorum procuratores super extractionem dictorum CCC kafficiorum panicii adacie et ordei nullum impedimentum vel obstaculum apponatis», guardando las

(14) Ibid.

(15) Ibid. Egidio Pérez (Petri) de Buysán era a la sazón el Guardasellos de la cancillería real.

(16) Ibid.

(17) ACA, Reg. 576, fol. 13 r. - v. Vid. Anexo Documental, II. 
cautelas idénticas a las ya expresadas más arriba, de no sacar cantidad mayor a la autorizada ni cualquier otro producto prohibido, sirviéndose de la presente licencia real y que vigile atentamente si el cargamento cerealístico llega a su destino prefijado y no a cualquier otro distinto. $Y$ haga cumplir todo cuanto queda ordenado escrupulosamente: «Et hoc nullatenus inmutetis" (18).

Queda constatado por una y otra carta el alto interés que se tomó el Infante $D$. Pedro de Aragón en socorrer puntualmente a la población de la villa de Alicante, azotada cruelmente por la plaga del hambre aquel año de 1333, con el aprovisionamiento de trescientos cahices de trigo, sorgo y cebada (19), equivalentes a la considerable suma de 600 hectólitros o quintales métricos, que arrojan la bella cifra global de 60.000 kilogramos de cereales de diferente calidad (20).

Si adoptamos por bueno el Censo poblacional de la villa de Alicante del 1409 , con motivo de aportación económica de las villas y lugares al matrimonio del Infante D. Martín I con Margarita de Prades, de 342 fuegos, con unos 1.700 habitantes (21), les corresponderían por casa y hogar $175 \mathrm{~kg}$. y por individuo $35 \mathrm{~kg}$. aproximadamente, cantidad de momento suficiente para matar el hambre y alejar provisionalmente el peligro amenazador.

La tercera carta del Infante D. Pedro de Aragón va dirigida al consejero, camerario real y bayle general del Reino de Valencia, D. Guillén Serani, para ordenarle que, ante la terrible carestía y escasez de los productos básicos de alimentación habitual, cuales son el arroz, higos, pasas o azebib y las algarrobas, y la consiguiente elevación extremada de sus precios, pasando a cobrarse la quartera de grano (1/5 fanega castellana) de 10-30 sueldos unidad, a la desorbitada cifra de 480 sueldos (22), debe tomar medidas en el asunto, impidiendo a toda costa la

(18) Ibid.

(19) Las clases de cereal citadas son las siguientes: Panizo 6 panicius; Mijo 6 adacsa, adacça, adacia, zahina, saina ó Sorghum Vulgare, panificable en las zonas norteñas para confeccionar la Boroña hasta la llegada del maíz, tras el descubrimiento del Nuevo Mundo; Cebada ú ordí = Hordeum. Vid. ALCOVER, DICC. CAT.-VALENC.-BALEAR, I, Palma de Mallorca 1978.

(20) Para la identificación de estas medidas de áridos en el Reino de Valencia y Corona de Aragón, v. DUFOURCQ, Ch. E., o.c., págs. 510-513 y 532-534.

(21) CUEVES GRANERO, A., "Aportación económica del Reino de Valencia al matrimonio de Martín I con Margarita de Prades", Estudios Medievales, I, Valencia, pág. 155; VILAR, J. Bta., HISTORIA DE LA CIUDAD DE ORIHUELA, t. III LOS SIGLOS XIV Y XV EN ORIHUELA, Orihuela 1977, pág. 18.

(22) DUFOURCQ, O.c., pág. 533, nota 81: “...el cafis de blat hi assolis 43 sous i més. Aquesta xifra és força inferior a la que dóna CAPMANY MEMORIAS, t. II, Apéndice pág. 122), per el periode de pesta $i$ fam que va viure Barcelona en aquell mateix any de 1333: 480 sous la quartera de blat!». 
exportación de los mismos fuera de la ciudad y Reino de Valencia, al objeto de paliar mejor males tran graves (23).

Guarda esta carta una relación muy estrecha con las dos anteriores, a las que sirve de contexto, manteniendo vigente la consabida prohibición de exportar las coses vedades y tanto más las mencionadas, tan de primera necesidad alimentaria, habida cuenta de la gravísima situación de penuria y escasez por que estaban pasando las gentes de todo el Reino de Valencia: "Urgens in dicto Regno frumenti et alterius bladi neccessitas, ex quo victualia sunt ibidem plus solito cariora" (24). $Y$ es una confirmación ulterior de la escasez y hambre que afligió por aquel final del año 1333 a todo el Reino de Valencia y a la villa concretamente de Alicante, de cuya constación documental son eco bien elocuente las dos cartas ya comentadas del propio Infante al bayle local de Cullera y al Bayle General, D. Guillén Serani, el destinatario también de esta tercera.

Queremos añadir finalmente una cuarta carta de D. Pedro de Aragón, dirigida a los oficiales encargados del control y vigilancia de aduanas y demás pasos fronterizos de la Corona, emanada también en Valencia, a 8 de enero de 1334, veinte días después de las tres anteriores (25), al objeto de que impidan la extracción por todos los medios de los consabidos productos prohibidos por cualquier punto o paso de sus fronteras. Les notifica a su vez que hagan excepción con el embajador del rey de Mallorca, Jaime III, ante la Santa Sede, D. Antonio de Galiana, quien goza de autorización real para sacar de aquel Reino balear un caballo de pelo claro y balzán del pie izquierdo trasero, con destino a la corte papal. Por todo ello les ordena que no le estorben la salida con el mismo ni pongan obstáculo alguno, durante el espacio de dos meses a partir de la fecha, ya que ése es el tiempo de duración de la cédula real, de suerte que concluidos los dos meses justos, no pueda sacarse ya del reino el caballo en cuestión: "Presentem non quam per duos menses a datatione huius littere in antea continue numerandos et non ultra valere volumus" (26).

Como puede apreciarse del contenido de esta última, cuyo texto íntegro transcribimos también al final, los caballos eran uno de los productos más codiciosos de la Corona y por ello asi mismo vedada su

(23) ACA, Reg. 576, fol. 14 v. Vid. Anexo Documental, III. Dada en Valencia, 26-XII-1333.

(24) Ibid.; DUFOURCQ, Ch. E.- GAUTIER, J.-DALCHE, HISTORIA ECONOMICA Y SOCIAL DE LA ESPAÑA CRISTIANA EN LA EDAD MEDIA, trad. esp. de la ed. fr. en París 1976, Barcelona 1983, págs. 223-224.

(25) ACA, Reg. 576, fol. 15 v. Vid. Anexo Documental, IV.

(26) Ibid. 
exportación, por cuanto se hallaba en guerra, como queda ya dicho, con la República de Géneva y el Reino Nazarí de Granada en aquel año preciso de 1333 y 1334. La prohibición de sacar cereales y demás productos panificables de primera necesidad se hacía igualmente indispensable por la escasez creciente de los mismos, por aquellas fechas, debido a las causas climáticas y las restantes bélicas, ya expresadas.

\section{CONCLUSION}

Nos es muy grato poder documentar un lugar más de la Corona de Aragón, concretamente la VILLA DE ALICANTE, como objeto singular de los primeros desvelos del joven Infante, a punto casi de ceñir la corona real, dos años tan sólo después, con el nombre de PEDRO IV DE ARAGON, ocupándose tan celosamente, como revelan elocuentes sus cartas en cuestión, de proporcionar el necesario avituallamiento a sus vecinos, ante la crisis alimentaria tan angustiosa y preocupante por que pasaban aquel fatídico año 1333 , tan faltos hasta del pan diario que llevarse a la boca.

Por todo ello hay que añadir a la ya larga lista de lugares que pasaron hambre aquel "primer año malo de 1333", en la Corona de Aragón, éste concreto de la villa de Alicante, en el Reino de Valencia, cuya necesidad alimentaria llegó a ser tan extrema, que escaseó el pan hasta no hallarse ya a la venta bocado alguno y verse por ello su población obligada al abandono de sus hogares y a emigrar a tierras mejores.

Providencial surgió entonces la figura del Infante D. Pedro, poniendo remedio a tanto mal, con el aprovisionamiento sustancioso de seiscientos quintales métricos de trigo-panizo, mijo y cebada, con que sofocar notoriamente aquel hambre tan cruel. 


\section{ANEXO DOCUMENTAL}

1333, diciembre 17. Valencia.

Carta del Infante $D$. Pedro de Aragón, primogénito de Alfonso IV, al bayle de Cullera, ordenándole que retire de inmediato la prohibición de sacar por su puerto trescientos cahices de grano con dirección a la villa de Alicante que, a consecuencia de la escasez de pan que llevarse a la boca, pasa por auténtica y grave necesidad, con peligro de tener que emigrar parte de su población, de no acudir en su remedio cuanto antes.

ACA, Reg. 576, fol. $6 \mathrm{r}$-v.

Infans Petrus etc. ffideli suo bajulo loci de Cullera salutem etc. In petitione nobis oblata/ pro parte hominum loci de Alacant vidimus et cernimus quod dicti homines volentes providere/ evidenti necessitati et penurie frumenti, quia propter anni presentis sterilitatem supraveniret/ loco predicto de Alacant, in quo panis coctus vix reperitur ut asseritur ad vendendum/, in loco de Aliazira (Alzira) emi fecerunt de consensu

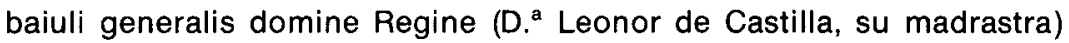
per eorum/ procuratorem ab emptoribus redituum dicte Regine, qui precipiuntur in eodem loco de/ Aliazira Centum Quinquaginta kafficia panicii et Centum quinquaginta aderis seu/adacie ad opus provisionis ipsorum hominum de Alacant, ne iidem homines/ fame compulsi haberent deserere locum ipsum et licet dicti homines de Alacant/ (fol. 6 v.) seu eius portator velint facere onerari dicta CCC kafficia panicii et adacie in portu/Cullarye pro ducendo ea ad dictum locum de Alacant, tamen vos (el bayle local de Cullera) pretextu inhibitionis/ in dicto loco de Cullera facte de non extrahendo ab eodem loco frumentum, pro/ dicta CCC kafficia panicii et adacie onerari in dicto portu et ab eodem extrahi inhibetis/. Quare ad humilem supplicationem per dictos homines de Alacant Nobis factam, ex causa/ necessitatis predicte, vobis dicimus et mandamus quatenus ipsis hominibus de Alacant vel eorum/ procuratori super extractionem dictorum CCC kafficiorum panicii et adacie nullum impe/ dimentum vel obstaculum apponatis. Quinimmo ipsa CCC kafficia panicii et adacie/ onerari in dicto portu et ab eodem extrahi per dictos homines de Alacant vel eorum procuratorem libere permittatis, inhibitione quarumqumque in aliquo non obstante,/ cavendo attente ne pretextu nostre concessionis huiusmodi inde ulla major/ frumenti seu aliarum rerum prohibitarum quantitas extrahatur, quodque dicti homines/ de Alacant seu eius portator sufficienter caveant in posse 
vestro quod dicta $\mathrm{CCC} /$ kafficia panicii et adacie ad alias partes preterquam ad ipsum locum de Alacant/ minime defferantur. Et hoc nullatenus inmutetis. Datum Valencie, XVI kalendas/ Januarij, anno domini M CCC XXX tertio/. Prescripta littera fuit restituta et lacerata et alia facta/ et expedita que infra registrata est in folio VII/Subsequenti/. Egidius Petri mandato domini Infantis facto/ per Petrum Jordani Durrer Expern/ cui fuit comissa super credentia (? Y per consilium domini Infantis/ (1).

1333, diciembre 17. Valencia.

Carta del Infante D. Pedro de Aragón al Bayle General del Reino de Valencia, $D$. Guillen Serani, ordenándole que, dada la carestía reinante de granos en la villa de Alicante, a raiz de la cosecha de cereales casi nula del año en curso, obligue al bayle local de Cullera a permitir la salida del puerto del cargamento que han podido recabar en Alzira los procuradores alicantinos, llegados allí con aquel cometido, ya que éste se escuda en la disposición real vigente de la prohibición de exportar cereales (Coses vedades) fuera del lugar, para impedir su transporte a la villa de Alicante.

ACA, Reg. 576, fol. 13 r.-v.

Infans Petrus etc. Dilecto suo Baiulo Regni Valencie Generali salutem etc. In petitione Nobis/ oblata pro parte hominum loci de Alacant vidimus contineri quod dicti homines volentes/ providere evidenti necessitati et penurie frumenti, quia propter anni presentis ste/ rilitatem supraveniret loco predicto de Alacant in quo panis coctus vix reperitur/, ut asseritur, ad vendendum, in loco de Aliazira emi fecerunt de consensu Bajuli/ Generalis domine Regine per eorum procuratorem ab emptoribus reddituum dicte/ Regine, qui percipiuntur in eodem loco de

(1) Es necesario subrayar que la escasez de cereales de aquel año 1333 llegó en Alicante a tal extremo que apenas se hallaba pan cocido a la venta, teniendo que arbitrar el concejo de la villa el envío de ciertos procuradores a Alcira, donde, con el asentimiento del Bayle General de la Reina, D. ${ }^{a}$ Leonor de Castilla y esposa de Alfonso IV de Aragón, Guillén Serani, en el Reino de Valencia, adquiriesen la suma de 150 cahices de panizo y 150 cahices más de mijo ó adacça, adacia o sorghum vulgare "con el que se hacían entonces las boroñas (panes de harina de mijo) antes de que el maíz viniera de América. En efecto, hoy el mijo apenas se emplea más que para pienso de las aves, mientras que en la Edad Media proporcionaba la mejor parte de la harina de pan en las zonas rurales" (COROMINAS, Johan, Diccionario crít., etimológico de la lengua castellana, vol. III, Madrid, 1974, pág. 374) para poder paliar la falta de tales productos de primera necesidad que se padecía por aquellas navidades en la región. 
Aliazira et etiam in loco de Cullera de/ decima eiusdem loci Quadraginta kafficia panicil et Ducenta dicta kafficia/ adorys seu adacie et Quinquaginta kafficia ordei ad opus provisionis ipsorum/ hominum de Alacant, ne iidem homines fame compulsi haberent deserere locum/ ipsum et licet dicti homines de Alacant seu eius portator velint facere onerarj dicta/ CCC kafficia panicii, adaci et ordei in portu Cullarie pro ducendo ea ad dictum/ locum de Alacant, tamen baiulus Cullarie pretextu inhibitionis in dicto loco de Cullera facte/ de non extrahendo ab eodem loco frumentum, predicta CCC kafficia panicij/ (fol. $13 \mathrm{v}$.), adacie et ordei onerari in dicto portu et ab eodem extrahi inhibet. Quare/ ad humilem supplicationem per dictos homines de Alacant Nobis factam ex causa/ necessitatis predicte, vobis dicimus et mandamus quatenus ipsis hominibus de Alacant vel/ eorum procuratores super extractionem dictorum $\mathrm{CCC}$ kafficiorum panicij, adacie et ordei/ nullum impedimentum vel obstaculum apponatis, quinimmo ipsa CCC kafficia/ panicii, adacie et ordei onerarj in dicto portu et ab eodem extrahi per dictos/ homines de Alacant vel eorum procuratores libere permittatis, inhibitione quacumque/ in aliquo non obstante. Cavendo attente ne pretextu nostre concessionis/ huiusmodi inde ulla major frumenti seu aliarum rerum prohibitarum quantitas/ extrahatur, quodque dicti homines de Alacant seu eius portator sufficienter/ caveant in posse vestro quod dicta CCC kafficia panicij, adacie et ordei ad/ alias partes preterquam ad ipsum locum de Alacant minime defferantur. Et hoc/ nullatenus inmutetis. Datum Valentie XVI kalendas Januarij, anno domini Millesimo/ tercentesimo trigesimo tertio/. Rodericus Didaci (Diaz) Cancellarij missit/ expedirj/ (1).

(1) Esta carta de contenido idéntico a la remitida al bayle local de Cullera, y de igual fecha, interesa al Bayle General del Reino de Valencia para que impida el celo excesivo de su subalterno de Cullera, al aplicar con rigor extremo la prohibición de productos y coses vedades del puerto de Cullera, vedando a los hombres de Alicante partir del mismo con el cargamento vital de vituallas para los vecinos de esta villa, en gravísimo aprieto de abastecimiento de pan, hasta el punto de tener que recurrir a la evacuación, de no acudir en socorro suyo. Es éste uno de los primeros bellos gestos del Infante D. Pedro, documentado puntualmente en los Registros, del mismo, con referencia a un rasgo tan singular y humano que transcurrió en la villa alicantina.

El encargado de la expedición de esta preciosa carta fue D. Rodrigo Díaz, Vicecanciller (y no Canciller) del Infante D. Pedro de Aragón, según nos lo certifica él mismo expresamente. 
1333, diciembre 26. Valencia.

Carta del Infante D. Pedro de Aragón al consejero real, camarlengo y Bayle General del Reino de Valencia, Guillén Serani, ordenándole que, ante la escasez y carestía de cereales por la pésima cosecha, casi nula, del año en curso, y la elevación desacostumbrada del precio de los mismos en el mercado, impida severamente la salida de la ciudad $y$ Reino de Valencia de los productos siguientes: arroz, higos, pasas o azebib y algarrobas, por el espacio de tiempo que se prolongue la referida penuria y al precio que estimen los jurados y hombres probos de Valencia oportuno.

ACA, Reg. 576, fol. 14V.

Infans Petrus etc. Dilecto nostro Guillelmo Serani dicti domini Regis Consilia/ rio et Camerario baiuloque Regni Valencie generali salutem etc. Urgens in dicto Regno/ frumenti et alterius bladi necessitas, ex quo victualia sunt ibidem plus solito $\mathrm{ca} /$ riora, nostrum quem ad dicti domini Regis et nostrorum commoda subditorum frecuen/ cius gerimus excitaverit animum et affectum, ut congruis sufulti remedijs/ ipsorum victualium penuriam possint commode subportare. Cum igitur in talibus/ casibus infrascripte res soleant plerisque sufragari, idcirco vobis dicimus et/ mandamus firmiter et expresse quatenus curetis districtius inhibere ne ri/ sum sive arrocium, ficus, pasule seu azebib et garrofie a dicta civitate vel/ Regno Valencie aliquatenus extrahatur. Quamquidem inhibitionem durare volu/ mus et jubemus per illud tempus quod juratis et probis hominibus dicte civitatis/ neccesarium videbitur et sub illis precijs de quibus eis visum fuerit expedire/.

Datum Valencie VII kalendas Januarij, anno domini Millesimo tercentesimo trigesimo tertio/.

Egidius Petri ex petitione propria/ (1).

(1) Es interesante relacionar esta orden del Infante D. Pedro con las cartas anteriores, a las que sirve de marco y contexto histórico, al prohibir la exportación de los productos referidos fuera de la ciudad y propio Reino de Valencia por aquel fin y principio de año, incluyendo aquellos cereales y leguminosas entre les "coses vedades" que no podian sacarse de su lugar de origen y producción, sin autorización regia expresa, a causa de la carestia tan grave de los mismos y terrible azote que supuso para los vecinos de Alicante y demás gentes de todo el Reino de Valencia. 
1334, enero 8. Valencia.

Carta del Infante $D$. Pedro de Aragón a los guardianes de los puntos de frontera, encargados de la vigilancia de mantener la prohibición de no sacar productos vedados o "coses vedades" de sus reinos, notificándoles la autorización real a favor del embajador del Rey de Mallorca, ante la Santa Sede y Curia Papal, D. Antonio de Galiana, para que pueda sacar del Reino de Mallorca un caballo de pelo claro y balzán o calzado del pie izquierdo trasero, con destino a la corte papal, sin que se le oponga por ellos el más leve reparo o dificultad. ACA, Reg. 576, fol. $15 \mathrm{v}$.

Infans Petrus etc. fidelibus suis custodibus passuum et rerum prohibitarum in/ confinibus nostre Regie constitutis et aliis officialibus Regiis atque nostris ad quos/ presentes pervenerint salutem etc. Noveritis Nos de speciali gracia concessisse Anto/ nio de Galiana, Illustris Regis Maioricarum in Curia Romana Procuratori/, quod possit extrahere seu extrahi facere de terra et dominatione dicti domini/ Regis quendam equum suum de pilo rucio, claro et balceranu de posteriori pede/ sinistro et eum ducere seu duci facere ad Papalem Curiam antedictam. Quare/ vobis et vestrum unicuique dicimus et mandamus quatenus prenominato Antonio vel/ cuicumque extrahenti pro eo equum predictum, super extractione ipsius a terra domini/ Regis predicti et nostra nullum obstaculum seu contrarium apponatis. Immo equum ipsum/ extrahi libere permittatis. Presentem non quam per duos menses a datatione huius littere/ inantea continue numerandos et non ultra valere volumus per illum ex vobis/ cui ultimo presentabitur in extractione dicti equi precipimus retineri. Datum Valencie/ sexto jdus Januarii, anno domini millesimo tercentesimo trigesimo tertio/.

Egidius Petri mandato domini Infantis facto/

Roderico Didaci Cancellario/. 